\title{
Allergie und Rheuma - Manifestation einer Grunderkrankung?
}

\author{
Allergische Erkrankungen sind gekennzeichnet durch eine \\ überschießende Produktion von Zytokinen aus $\mathrm{TH}_{2}$-Zellen. Eine \\ einseitige Verschiebung der Immunantwort in Richtung $\mathrm{TH}_{2}$-Zellen \\ war deshalb bisher eine Erklärung für die Entstehung von Allergien. \\ Doch weitaus komplexere Mechanismen werden diskutiert.
}

mmunerkrankungen nehmen in der ganzen westlichen Welt dramatisch $\mathrm{zu}$ - nicht nur allergische Erkrankungen wie Asthma, Rhinitis oder Ekzeme, sondern auch Typ-1-Diabetes, Psoriasis oder Rheuma. Letztere werden durch eine $\mathrm{TH}_{1}$-vermittelte Autoimmunreaktion hervorgerufen.

Als Ursache für die Inzidenzzunahme beider Formenkreise von Erkrankungen werden veränderte Umweltbedingungen angenommen. So wird die als Hygienehypothese bekannt gewor- dene Vermutung, dass eine verminderte Auseinandersetzung mit Infektionen im frühen Kindesalter die $\mathrm{TH}_{2}$-vermittelte Atopie fördern könnte, auch beim $\mathrm{TH}_{1}$-vermittelten Typ-1-Diabetes diskutiert. Diese Erkrankung tritt ebenfalls gehäuft in kleinen Familien und bei Erstgeborenen auf.

Die Annahme, dass $\mathrm{TH}_{1}{ }^{-}$und $\mathrm{TH}_{2}$-vermittelte Krankheiten möglicherweise eine gemeinsame Ursache haben, wird durch die Auswertung der Daten von 252.538 Patienten des

schottischen CMR (Continous Morbidity Recording)-Projektes gestützt. Danach ergab sich ein signifikant erhöhtes Risiko für Patienten mit einer atopischen Genese, zusätzlich eine $\mathrm{TH}_{1}$-vermittelte Autoimmunerkrankung zu entwickeln. Umgekehrt wurden signifikant häufiger Patienten mit einem Ekzem oder allergischer Rhinitis vorstellig, die eine $\mathrm{TH}_{1}$-vermittelte Erkrankung in der Anamnese hatten.

\section{Fazit}

$\mathrm{TH}_{1}$ - und $\mathrm{TH}_{2}$-vermittelte Immunerkrankungen schließen sich nicht gegenseitig aus. Vielmehr treten sie nicht selten gemeinsam auf und scheinen möglicherweise durch identische Umweltfaktoren hervorgerufen zu werden. $b k$

Simpson CR et al. Coincidence of immune-mediated diseases driven by $\mathrm{Th}_{1}$ and $T h_{2}$ subsets suggests a common aetiology. Clin Exp All 2002; 32: 37-42

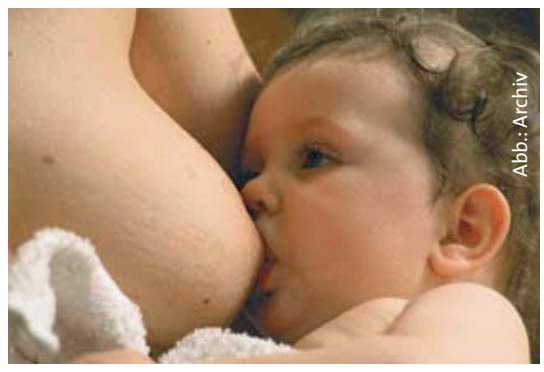

Bei Milch geht nichts über das Original.

n 20 Kindern zwischen 1 Monat und 9 Jahren mit positivem Nahrungsmittel-Provokationstest auf Kuhmilch sollte die Reaktion auf verschiedene Milch-Substitutionsprodukte verglichen werden. Es handelte sich dabei um ein stark hydrolisiertes und ein teilweise hydrolysiertes Molkeprodukt, ein stark hydrolysiertes Caseinsubstitut sowie eine Aminosäure-Zubereitung.

Im Haut-Prick-Test reagierten gegen das stark hydrolisierte Substitut $15 \%$, gegen das teilweise hydrolisierte Produkt 55\%, gegen hydrolisiertes Casein $10 \%$ und gegen das AminosäureDerivat $5 \%$ der Kinder. Bei der IgE-Bestimmung zeigten 90\% Kuhmilch-spezifische IgE-Antikörper, 65\% Antikörper gegen Casein, 60\% Antikörper gegen Laktoglobulin und 55\% Antikör- per gegen Laktalbumin. Hinsichtlich der Substitute hatten 10\% der Kinder Antikörper gegen das stark hydrolisierte Molkeprodukt, jeweils 5\% gegen das teilweise hydrolisierte Molkesubstitut und gegen das hydrolisierte Casein entwickelt. Gegen das Aminosäure-Derviat wurden keine Antikörper gefunden.

Im Nahrungsmittel-Provokationstest reagierten 5 von 17 Kindern auf das starke Hydrolysat, 3 von 16 Kindern auf das teilweise hydrolisierte Molkeprodukt, 2 von 10 Kindern auf die Aminosäurezubereitung und nur 1 von 16 Kindern auf das Caseinhydrolysat. Bei den verschiedenen Hydrolysaten kam es vereinzelt (insgesamt in 6 Fällen) zu Sofortreaktionen wie Erbrechen, Durchfall, Urtikaria, Angioödem, Dyspnoe und Blutdruckabfall.

\section{Fazit}

Keines der untersuchten Kuhmilchsubstitute konnte in dieser Studie in jedem Falle eine allergische Reaktion verhindern. Die Autoren empfehlen bei der Gabe von Hydrolysaten eine klinische Beobachtungsphase, da in der Studie auch Sofortreaktionen beobachtet wurden. Das Attribut "hypoallergen“ möchten die Autoren nach diesen Ergebnissen nur den stark hydrolysierten Casein-Produkten zuerkennen. romw

Caffarelli C et al. Determination of allergenicity to three cow's milk hydrolysates and an aminoacid derived formula in children with cow's milk allergy. Clin Exp All 2002, 32: 74-9 